\title{
Risk factors detection in chronic thromboembolic pulmonary hypertension, a tool for risk quantification?
}

\author{
Bohacekova $\mathrm{M}^{1}$, Kaldararova $\mathrm{M}^{1}$, Valkovicova $\mathrm{T}^{1}$, Remkova $\mathrm{A}^{2}$, Vesely $\mathrm{J}^{3}$, Simkova $\mathrm{I}^{1}$ \\ Department of Cardiology and Angiology, Faculty of Medicine, Slovak Medical University and National \\ Institute of Cardiovascular Diseases, Bratislava, Slovakia. marcela.bohacekova@gmail.com
}

\begin{abstract}
BACKGROUND: Chronic thromboembolic pulmonary hypertension (CTEPH) is characterized by chronic thromboembolic obstruction in the pulmonary bed. The definitive pathogenesis remains incompletely explained, although multiple risk factors of CTEPH have been identified.

The purpose of the study was to evaluate the risk profile of patients with CTEPH and the representativeness of risk factors, identify possible new CTEPH risk factors and specify the epidemiology of CTEPH in our country. METHODS: In 81 patients with CTEPH, well-known risk factors were analyzed, and a detailed analysis of selected hematological parameters was investigated at a specialized hematology laboratory.

RESULTS: CTEPH risk factors were identified as follows: pulmonary embolism (PE), deep vein thrombosis (DVT), thyreopathy, blood type other than "0", inflammatory bowel disease, malignancy, splenectomy, pacemaker. When compared to healthy controls, the following was observed: a significant decrease in platelet count, higher mean platelet volume, higher spontaneous platelet aggregation, increase in von Willebrand factor and fibrinogen. The median of risk factors representativeness was 3 (PE, DVT, blood type other than "0"). The prevalence of CTEPH in adult population in our country is estimated to be 1.8 per 100,000 inhabitants.

CONCLUSION: In the study we confirmed multiple established risk factors of CTEPH, set their representativeness, identified some platelet abnormalities which could be a potential new risk marker and specified the prevalence of CTEPH (Tab. 5, Ref. 35). Text in PDF www.elis.sk.

KEY WORDS: chronic thromboembolic pulmonary hypertension, risk factors, coagulation.
\end{abstract}

\section{Introduction}

Chronic thromboembolic pulmonary hypertension (CTEPH) is a progressive chronic disease defined as the persistence of pulmonary hypertension $(\mathrm{PH})$ (mean pulmonary artery pressure (mPAP) $\geq 25 \mathrm{~mm} \mathrm{Hg}$ ) after a single or recurrent pulmonary embolism (PE) event. CTEPH results from persistence of thrombotic obstructions in the pulmonary vasculature in the presence of obliterative vascular remodeling despite effective anticoagulation treatment for at least 3 months $(1,2)$. The current understanding of the CTEPH pathophysiology is discussed as well as the concept of misguided thrombus resolution (3). Up-to-date data suggest that CTEPH does not result from traditional risk factors of venous thromboembolism (VTE) (4). However, there is a list of well-known risk factors contributing to the development of CTEPH divided in three

${ }^{1}$ Department of Cardiology and Angiology, Faculty of Medicine, Slovak Medical University and National Institute of Cardiovascular Diseases, Bratislava, Slovakia, ${ }^{2}$ Department of Internal Medicine, Faculty of Medicine, Slovak Medical University, Bratislava, Slovakia, and ${ }^{3}$ Department of Nuclear Medicine, Faculty of Medicine, Comenius University, Faculty of Medicine, Slovak Medical University, St. Elizabeth Cancer Institute, Faculty of Medicine, Comenius University, Bratislava, Slovakia

Address for correspondence: M. Bohacekova, MD, Department of Cardiology and Angiology, Faculty of Medicine, Slovak Medical University and National Institute of Cardiovascular Diseases, Pod Krasnou horkou 1, SK-833 48 Bratislava, Slovakia. Phone: +421.2.59320271 groups based on known medical conditions (5) in association with symptomatic PE and laboratory risk factors (6).

Some medical conditions well known for years as risk factors for CTEPH, as well as validated by recent international registries include PE, deep vein thrombosis (DVT), ventriculoatrial shunts, indwelling venous catheters and leads, splenectomy history, thyreopathy and thyroid replacement therapy, chronic inflammatory disorders such as inflammatory bowel disease (IBD) and osteomyelitis, non-“0”" blood group, lipoprotein A, and history of malignancy or myeloproliferative disorders $(3,7,8)$.

Risk factors for CTEPH associated with PE include the idiopathic nature of $\mathrm{PE}$, recurrence of $\mathrm{PE}$ as well as large perfusion defects, younger age, proximal $\mathrm{PE}$, and systolic pulmonary artery pressure $>50 \mathrm{~mm} \mathrm{Hg}$ at diagnosis $(9,10)$.

Some laboratory risk factors as elevated plasma concentrations of the coagulation factor VIII, lupus anticoagulant (LA) and antiphospholipid antibodies (APA), von Willebrand factor (vWF), plasminogen activator inhibitor type 1 (PAI-1) and fibrinogen (10, 11) have been found in CTEPH population.

Understanding the pathophysiology and early identification of potential risk factors can help identify at-risk patients, change the treatment paradigm to a preventative strategy and thus improve the prognosis $(1,3)$.

The purpose of the study was to evaluate the risk profile of patients with CTEPH, set the representativeness of risk factors managed at our institution, specify the epidemiology of CTEPH 


\section{$577-582$}

in our adult population and identify new potential CTEPH risk factors.

\section{Patients and methods}

Study population consisted of 81 patients (30 females/51 males) with CTEPH confirmed by accepted diagnostic algorithm at our institution between October 1998 and December 2014. Their mean age was $60.5 \pm 11.9$ and the interval from onset of symptoms to cardiac catheterization was $32.6 \pm 25.9$ months.

Other baseline characteristics as values of $\operatorname{mPAP}(48.6 \pm 12.0$ $\mathrm{mm} \mathrm{Hg}$ ), pulmonary vascular resistance (PVR: $9.2 \pm 4.6$ W.u.), cardiac index $\left(\mathrm{CI}: 2.3 \pm 0.6 \mathrm{~L} \cdot \mathrm{min}^{-1} \cdot \mathrm{m}^{-2}\right)$, mean right atrial pressure (mRAP: $9.8 \pm 4.2 \mathrm{~mm} \mathrm{Hg}$ ), arterial oxygen tension $\left(\mathrm{PaO}_{2}: 62.1\right.$ \pm 8.4 torr), as well as body mass index (BMI: $27.4 \pm 4.7 \mathrm{~kg} / \mathrm{m}^{2}$ ), clinical severity according to NYHA (New York Heart Association) functional class $(2.8 \pm 0.5)$ and exercise capacity according to 6 -minute walking test (6MWT: $313.4 \pm 134.3 \mathrm{~m}$ ) at the time of diagnosis are seen in the Table 1 . If we simplify the baseline characteristics (hemodynamic and clinical), a typical patient with CTEPH suffers from severe PH with low exercise tolerance and subjectively considerable dyspnea.

Almost $50 \%$ of patients $(n=40)$ underwent surgical therapy (PEA) and 18 patents were/are treated by specific pharmacotherapy of $\mathrm{PH}$. Prior to the admission to our institution, all patients were effectively anticoagulated with vitamin $\mathrm{K}$ antagonist at least for three months (e.g. to a target INR of 3.0). The diagnostic algorithm we performed consisted of transthoracic echocardiography (TTE), V/P scan (at least one segmental or wider perfusion defect with normal ventilation), CT pulmonary angiography (chronic thromboembolic signs: organized thrombus occluding the pulmonary arteries completely, and/or else eccentrically and/or concentrically, and/or a mosaic perfusion picture in the parenchyma with reduced vascular calibration in distal regions and pulmonary artery dilatation), DSA pulmonary angiography (abrupt branch tapering, complete vessel occlusion, luminal irregularities caused by mural clot, pouch-like regions related to occlusive or sub-occlusive

Tab. 1. Patient characteristics $(n=81)$.

Age (years)
Gender $(\mathrm{F} / \mathrm{M})$
BMI
Duration of symptoms (months)
NYHA/WHO functional class
6MWT $(\mathrm{m})$
mRAP $(\mathrm{mm} \mathrm{Hg})$
mPAP $\left(\mathrm{mm} \mathrm{Hg}^{-1}\right)$
CI $\left(\mathrm{L} \cdot \mathrm{min}^{-1} \cdot \mathrm{m}^{-2}\right)$
PVR (dyn $\cdot \mathrm{s}^{-5} \mathrm{~cm}^{-5}$
PaO $($ torr $)$
Comorbidity
PEA (n)

$$
\begin{gathered}
60.5 \pm 11.9 \\
30 / 51 \\
27.4 \pm 4.7 \\
32.6 \pm 25.9 \\
2.8 \pm 0.5 \\
313.4 \pm 134.3 \\
9.8 \pm 4.2 \\
48.6 \pm 12.0 \\
2.3 \pm 0.6 \\
9.2 \pm 4.6 \\
62.1 \pm 8.4 \\
78.4 \\
40
\end{gathered}
$$$$
18
$$

Spec. FTx (n)

Data given as mean $\pm \mathrm{SD}, \%$ or $\mathrm{n}$. CI - cardiac index; DVT - deep vein thrombosis; Spec. FTx - specific fakmacotherapy; mPAP - mean pulmonary arterial pressure; mPAR - mean right atrial pressure; PVR - pulmonary vascular resistance; NYHA/ WHO - New York Heart Association/World Health Organization; PEA - pulmonary endartherectomy. 6MWT - 6-minute walking test, BMI - body mass index thrombi, and webs or bands that may cause branch narrowing and post-stenotic dilatation) as the method of diagnostic reference and assessing the eligibility of surgery and by right heart catheterization ( $\mathrm{mPAP} \geq 25 \mathrm{~mm} \mathrm{Hg}$ and a pulmonary capillary wedge pressure $(\mathrm{PCWP}) \leq 15 \mathrm{~mm} \mathrm{Hg}$, CI by thermo-dilution method with Swan-Ganz Catheter and / or by Fick method).

In all patients, we analyzed medical history, clinical signs and symptoms and concomitant diseases, which were obtained from his/her health card and during the personal interview. We focused on well-known CTEPH risk factors: PE, recurrent PE, idiopathic PE, DVT, malignancy, chronic inflammatory conditions, including osteomyelitis, IBD, pacemaker leads, thyreopathy, splenectomy, autoimmune disease, and blood type other than " 0 ".

At specialized hematology laboratory a detailed analysis of blood coagulation parameters, thrombocytes count, thrombocytes aggregation assessment and endothelial dysfunction evaluation was performed. Platelet aggregation was investigated by light transmission aggregometry without stimulation (i.e. spontaneous aggregation). The vWF and PAI-1 were used as indicators of endothelial dysfunction and/or damage. Selected platelet, endothelial and clotting parameters were investigated in 36 patients with confirmed CTEPH, as well as in 32 healthy subjects representing a control group.

\section{Statistical analysis}

Student $t$-test or the one-way analysis of variance test for normally distributed data and the Wilcoxon test for non-parametric data were used. In case of nominal data, contingence tables were used. These comparisons and logistic regression analyses were performed using JMP version 5.0.1 software (SAS Institute Inc, Cary, NC, USA) and Windows Microsoft Excel 2007. Results are expressed as mean \pm standard deviation for continuous variables and as a number and percentage for categorical variables. The differences were considered statistically significant at a significance level of $\mathrm{p}<0.05$.

\section{Results}

Table 2 shows known CTEPH risk factors. In total, $79 \%$ of all patients were previously diagnosed with PE, only $9.9 \%$ were treated with thrombolytic therapy. Previous DVT was observed in

Tab. 2. Risk factors $(n=81)$ (in \%).

\begin{tabular}{lc}
\hline Inherited thrombophylia & 22.2 \\
Pulmonary embolism & 79.0 \\
Recurrent PE & 28.4 \\
Idiopathic PE & 19.8 \\
DVT & 59.3 \\
Thrombolytic therapy & 9.9 \\
Tyreopathy with a thyroid replacement therapy & 19.8 \\
Blood type other than "0" & 71.6 \\
Malignity & 6.2 \\
Morbus Crohn/ Ulcerative colitis & 6.2 \\
Splenectomy & 2.5 \\
Pacemaker electrode & 2.4 \\
Coagulation abnormality & 28.9 \\
\hline Data given as mean \pm SD, \% or $n . p<0.05$. DVT - deep vein thrombosis, PE - pul- \\
monary embolism.
\end{tabular}


Tab. 3. Hematological risk factors $(n=19)$.

\begin{tabular}{lccc}
\hline & CTEPH & Control group & $\mathrm{p}$ \\
\hline Trombocytes count $\left(10^{9} / \mathrm{l}\right)$ & $216 \pm 56$ & $261 \pm 60$ & $<0.01$ \\
MPV (fl) & $11.2 \pm 0.9$ & $9.9 \pm 0.7$ & $<0.001$ \\
SPA (\%) & $10.9 \pm 4.3$ & $8.4 \pm 6.2$ & $<0.05$ \\
vWf: Ac (\%) & $184.2 \pm 28.6$ & $122 \pm 37.3$ & $<0.001$ \\
vWf: Ag (\%) & $186.5 \pm 23$ & $124 \pm 36.4$ & $<0.001$ \\
PAI-1 (U/mL) & $2.8 \pm 1.6$ & $2 \pm 1.4$ & $=0.05$ \\
Fibrinogen (g/L) & $3.8 \pm 0.8$ & $3.1 \pm 0.6$ & $<0.001$ \\
Faktor VIII (\%) & $158.2 \pm 30.7$ & $129.6 \pm 31.5$ & $<0.001$ \\
\hline
\end{tabular}

Data given as mean \pm SD, \% or n. $\mathrm{p}<0.05$. MPV - mean platelet volume, SPA spontaneous platelet aggregation, vWf - von Willebrand factor, vWf: Ac - activity and vWf: Ag - antigen, PAI-1 - plasminogen activator inhibitor type 1

Tab. 4. Count of CTEPH risk factors.

\begin{tabular}{ccc}
\hline Count of risk factors $(\mathrm{n})$ & Patients $(\mathrm{n})$ & Patients $(\%)$ \\
\hline 0 & 2 & 2.5 \\
1 & 9 & 11.1 \\
2 & 20 & 24.7 \\
3 & 31 & 38.3 \\
4 & 12 & 14.8 \\
5 & 6 & 7.4 \\
6 & 1 & 1.2 \\
\hline Median: 3 & & \\
\hline
\end{tabular}

Tab. 5. Triple combination of CTEPH risk factors.

\begin{tabular}{cccc}
\hline $\begin{array}{l}\text { Count of risk } \\
\text { factors }(\mathrm{n})\end{array}$ & $\begin{array}{c}\text { Patients } \\
(\mathrm{n})\end{array}$ & $\begin{array}{c}\text { Triple combination } \\
\text { of RF }(\mathrm{n})\end{array}$ & $\begin{array}{c}\text { Triple combination } \\
\text { of RF (\%) }\end{array}$ \\
\hline 3 & 31 & 15 & 48.4 \\
4 & 12 & 9 & 75 \\
5 & 6 & 4 & 66.7 \\
6 & 1 & 1 & 100 \\
\hline SUM & 50 & 29 & 58 \\
\hline
\end{tabular}

Triple combination: PE (pulmonary embolism), DVT (deep vein thrombosis), and blood type other than " 0 "

$59.3 \%$ of all cases and an idiopathic PE was confirmed for $19.8 \%$ of all CTEPH patients.

Additionally, more than $19 \%$ of all patients have a history of thyreopathy with replacement therapy, more than $71 \%$ have blood type other than " 0 ", an IBD have $6.2 \%$ and the same count of patients with CTEPH have a history of malignancy. From other expected risk factors: splenectomy and pacemaker leads were only in $2.5 \%$ of all our patients.

Hematological risk factors in comparison with a healthy control group are shown in Table 3. While the thrombocytes count is significantly lower in patients with CTEPH $\left(216 \pm 5610^{9} / 1\right.$ vs 261 $\left.\pm 6010^{9} / 1\right)$, mean platelet volume (MPV) values are significantly higher $(11.2 \pm 0.9 \mathrm{fl}$ vs $9.9 \pm 0.7 \mathrm{fl})$. Also, spontaneous platelet aggregation (SPA) was significantly higher in the CTEPH group $(10.9 \pm 4.3 \%$ vs $8.4 \pm 6.2 \%)$ as well as all other values, namely vWf activity (vWf:Ac), vWf antigen (vWf:Ag), fibrinogen and factor VIII, except for PAI-1 which was also higher in CTEPH group, but not significantly. Values of LA/APA were not increased.

Tables 4 and 5 demonstrate the occurrence of CTEPH risk factors with median of three risk factors. Majority of our patients (77.8 \%) had 2-4 of above-mentioned risk factors. If the patient had only one risk factor, then it was either PE/DVT or blood type other than " 0 ". In case of two risk factors, these were always in combination as follows: PE/DVT/ blood type other than " 0 "/ inherited thrombophilia/thyreopathy with a thyroid replacement therapy. In case of three risk factors the most frequent combination was PE and/or recurrent PE, DVT, blood type other than " 0 ". This triple combination was found in $58 \%$ of CTEPH patients with three or more risk factors (Tab. 5).

The prevalence of CTEPH in our adult population is estimated to be 1.8 per 100,000 inhabitants.

\section{Discussion}

In our study group of $81 \mathrm{CTEPH}$ patients coming from the entire nation, we found that previous PE, DVT and blood type other than " 0 " are the most frequent well-accepted risk factors for CTEPH development. One-third to one-fifth of them suffered from recurrent and idiopathic $\mathrm{PE}$, thyreopathy, or had coagulation abnormality and inherited thrombophilia. In less than $10 \%$, malignancy, bowel disease, permanent cardiostimulation, splenectomy or autoimmune disease as risk factor could be detected. Majority of established risk factors found in our study (PE, DVT, thyreopathy with thyroid replacement therapy, non-"0" blood groups, significantly increased vWf, fibrinogen and factor VIII) strongly coordinate with world-wide observation. In case of splenectomy, we had only $2.5 \%$ which is a relatively small difference and we could explain it with a possibly lower indication of splenectomy in our region. Nowadays, the incidence of splenectomy (splenectomies for treatment of hematologic malignancies and cytopenia, as well as incidental and iatrogenic splenectomies) has decreased dramatically. IBD in our study was presented equally to all studies which refer IBD incidence in CTEPH patients. In our study, cancer as a possible risk factor in CTEPH was only about a half of those presented in other studies. Increased levels of LA/APA were not found in our study. These two markers represent an exception from all examined parameters. The variety in LA/APA values could be explained with a small number of patients who underwent a hematological screening. Moreover, we found out that the SPA value is significantly higher in patients with CTEPH. Cancer is a serious condition and it is possible that patients suffering from malignity die before they are sent to a specialized center.

According to the well-accepted embolic hypothesis of CTEPH, the disease starts with an occlusion of proximal pulmonary arteries due to PE and initial PH which leads to general vascular remodeling and distal pulmonary arteriopathy, so-called microvascular disease. The early recognition of VTE can help detect patients at risk. Ultrasonography evidence of DVT was found in $56.1 \%$ (10) of patients with CTEPH in an International Prospective Registry with 456 CTEPH patients. In our study population of 81 patients, a very similar result was achieved (59.3\%).

Many studies investigated the incidence of PE in patients with CTEPH, and based on their results, about half of these patients had no history of PE (8). While these studies indicate PE incidence between $42 \%$ (13), $52 \%$ (14), $63 \%, 74.8 \%$ (10) and $80 \%$ (5), $25.2 \%$, respectively (15), our study showed an outcome of $79 \%$ in all CTEPH cases. It does not mean that we have better diagnostic methods for CTEPH in our country, but it evokes an idea that 


\section{7-582}

some patients with symptoms of CTEPH after PE are referred for evaluation by a cardiologist to CTEPH center very late.

Some known risk factors were found in studies as thyreopathy with a thyroid replacement therapy, namely in almost $20 \%$ of all patients (10). The pathophysiology of CTEPH under this condition is not well explained. Patients with hypothyroidism are associated with having an increased risk of thrombosis. The treatment with levothyroxine increases the von vWf levels and shortens in vitro platelet plug formation. It is unknown whether the treatment of the disease or the disease itself is responsible for the increased incidence of CTEPH $(16,17)$. In our CTEPH patients, the thyreopathy with a thyroid replacement therapy was presented in 19.8 $\%$, which strongly correlates with up-to-date knowledge.

In our study, splenectomy was found to occur only in $2.5 \%$ of all CTEPH patients which stands in contrast to other studies, where $3.4-5.5 \%$ of patients with CTEPH had undergone a previous splenectomy $(10,18)$. The association between splenectomy and CTEPH may be explained by the presence of abnormal erythrocytes that would otherwise be filtered out of the blood by the spleen (19). The formation of thromboembolic material is due to activation of coagulation caused by an abnormal expression of phosphatidylserine on the surface of erythrocytes. The other explanation of increased risk for CTEPH following a splenectomy could be the reactive thrombocytosis. After having the splenectomy, an increase in circulating microparticles and negatively charged phospholipids is enhanced by experimental thrombus induction $(18,19,20)$. One of the consequences of having a splenectomy and presumably also the cause of infection and cancer may lie in increased plasma levels of microparticles and anionic phospholipids, and may explain the abnormal tendency to thrombosis in CTEPH (20).

Pathological remodeling of pulmonary arteries is triggered by "inflammatory thrombosis" caused by coagulation disturbances, sticky red blood cells, uncleavable fibrinogen. This can be evoked by immunologic, inflammatory, or infectious conditions $(21,22)$. Mildly increased C-reactive protein levels in patients without concomitant leukocytosis are more likely correlated with an inflammatory state caused by chronic heart failure as a consequence of CTEPH than due to an active bacterial infection (23). From these data, we can infer that bacterial DNA within chronic thrombi represents evidence of some previous infection. There was a significant reduction in the appearance of macrophages essential for thrombus recanalization in infected murine thrombi $(14,18)$.

The IBD incidence in registries varies between 2.8-14\%; in our study we found a similar count, namely $6 \%$. IBD did not reach statistical significance in current studies. Overall $10 \%$ of patients but no health control had a history of osteomyelitis or IBD (24). Interestingly, $14 \%$ of CTEPH patients with no history of VTE have suffered from osteomyelitis or IBD. In comparison with non-thromboembolic pulmonary hypertension, 12 patients were affected in the CTEPH group ( $1.2 \%$ vs $2.8 \%$, respectively).

It is well known that patients with cancer are at increased risk of thrombosis and VTE (well-accepted paraneoplastic syndrome). Since more than $12 \%$ of patients with CTEPH were reported to have a history of malignancy, the evidence that malignancy and/or the treatment-related factors could be a risk factor for CTEPH is strong $(18,25)$. In our study, the history of malignancy was present in $6.2 \%$ of all patients.

In previous studies, $77 \%$ of patients with CTEPH were found to have non-"0" blood groups, while in ours it was more than $71 \%$. Hence, we can state that it correlates with the knowledge we have(14).

It is accepted, that traditional risk factors of VTE do not work in CTEPH (10). However, an elevation in some traditional hematological laboratory factors (elevated plasma concentrations of factor VIII, vWf:Ac, vWf:Ag, PAI-1) could be detected also in CTEPH (11).

Increased levels of factor VIII, which is an accepted risk factor for recurrent PE, have been shown to be present in patients with CTEPH in comparison with healthy subjects (26). Abnormalities in coagulation and fibrinolysis pathways have been associated in the minority of CTEPH patients with most prevalent ones being LA and APA. Thrombophilia studies have shown that LA may be found in $10 \%$ of CTEPH patients, and $20 \%$ carry LA/APA or both $(21,27,28)$. Contrary to other studies, LA/APA levels in our CTEPH patients were not increased. In our study, we also focused on hematological parameters, while significantly higher levels of factor VIII and vWF were demonstrated. This phenomenon correlates with the current knowledge of the issue. An elevated level of factor VIII was identified as a risk factor for CTEPH in a study of 122 patients compared with 82 healthy controls (26). The vWF is secreted into plasma from endothelial cells and plays a crucial role in platelet adhesion and aggregation $(11,29)$. The level of vWF was also elevated in these patients compared with the control group.

CTEPH may occur not only due to a complex interaction between thrombotic/thrombolytic processes like in classic VTE, but also due to angiogenic cellular remodeling of organized thrombi as well as failed fibrinolysis (30). In endothelial cells lining, PAI-1, an inhibitor of fibrinolysis, is increased in conjunction with low tissue-type plasminogen activator antigen levels, thus suggesting that the endothelium interaction with thrombus may be phenotypically altered (31). Moreover, in neovessels within CTEPH thrombi, an elevated expression of type PAI-1 has been found, which may influence the resolution of the thrombus. However, when PAI-1 was measured in plasma of CTEPH patients, no difference was seen in the relative levels of plasminogen activator and PAI-1 compared with healthy controls. This fact may suggest higher PAI-1 expression in the CTEPH thrombus. The elevation of PAI-1, fibrinogen, factor VIII, vWF: Ag, vWF: Ac can be also as the consequence of damage to the vascular endothelium and/or a response to inflammation. CTEPH-associated dysfibrinogenemias may be responsible for incomplete clot resolution in CTEPH patients (31). In our study we found significantly higher values of these mentioned hematological parameters in comparison with healthy control, except for PAI-1 which was also higher in CTEPH group, but not significantly.

Moreover, we found a higher SPA together with low platelet count and increased MPV in CTEPH patients. These platelet abnormalities represent a specific platelet behavior in CTEPH. This is in correlation with the idea of increased platelet destruction $(14,32)$. This finding may reflect a higher platelet turnover due to chronic process of platelet activation, consumption or destruction, in which the production of new (younger, bigger and more potent) platelets 
is not sufficient and may lead to thrombocytopenia (33). These platelet abnormalities could pose a potential new risk marker for CTEPH, the possibility of which has not been yet published in literature. Recent studies mention the role of platelet in CTEPH but no study has yet considered increased SPA to be a risk factor ofCTEPH.

The largest study analyzing CTEPH risk factors was prospectively carried out on 456 patients, including 9 patients with CTEPH from our country. Our study brings an original view on $81 \mathrm{CTEPH}$ patients in our country, but with limitation of study in prospective/ retrospective manner. This study analyses all consecutive patients with CTEPH at a single institution (one center for the whole country, i.e. for 4.4-million adult population). This study, similarly to a registry, reflects the epidemiology of this rare disease.

The guidelines do not recommend a methodical screening of patients after PE in case of CTEPH development. The history of $\mathrm{PE}$ seems to be the strongest risk factor of CTEPH. Therefore, the screening of other established risk factors of CTEPH is also not recommended. Nevertheless, it is necessary for understanding the pathophysiology of the development, progression of CTEPH in order to implement effective therapeutic and diagnostic strategies to decrease mortality rates (34). Studies show that the long-term prognosis of operated patients is better than the outcome of not operated patients (35).

The multivariate of risk factors limit us to detect correctly patients at-risk or to quantify the risk. We tried to set the most frequent combination of established risk factors in our patients, and found out the most frequent risk factors as well as their strongest combination. In patients with CTEPH presenting with one risk factor, we found either PE, DVT or non-"0" blood groups as the most frequent risk factor. When the patient had two risk factors of CTEPH, these were always one of double combination as follows: PE, DVT, non-“0” blood groups, inherited thrombophilia and thyreopathy. Overall, $70 \%$ of those patients with two risk factors had a combination PE and DVT, or PE and non-" 0 " blood groups. Interestingly, when the patient had three risk factors, everyone presented with PE and/or DVT. In case four or more risk factors were concurrent, it was always non " 0 " blood group and PE and/or DVT in combination with one of the above mentioned risk factors. The most frequent triple combination of risk factors was $\mathrm{PE}$ and/or recurrent PE, DVT, blood type other than " 0 ". Based on the occurrence of CTEPH risk factors, we are not able to predict the probability of CTEPH development. The triple combination seems to be the strongest one, which may represent a facilitating moment in the development of this disease.

Interestingly enough, all patients who underwent splenectomy, had increased SPA. That evokes and partially confirms the hypothesis that platelet dysfunction can play an important role in CTEPH pathogenesis, and more research in this way is needed.

In fact, this study brings a valuable asset from the epidemiologic point of view because all CTEPH patients in our country are treated at our institution. Thus, this single center study can be considered to be an epidemiologic study as well, and brings important reference to the epidemiology of this rare disease. As a result, we define, that the prevalence of this rare disease in adult population from our country is 1.8 per 100,000 inhabitants.
The real prevalence is unfortunately unidentified and difficult to determine and may be significantly underestimated. CTEPH is often misdiagnosed due to nonspecific symptoms and variable disease course, just because acute PE can occur without symptoms, and may often be overlooked in practice. This is the reason for systematic evaluation of the population at-risk for CTEPH and raising the awareness of the issue in medical practice.

\section{Conclusion}

Understanding the pathophysiology and early identification of potential risk factors will help identify at-risk patients, change the treatment paradigm into preventative strategy and improve the prognosis. Several risk factors of CTEPH (history of PE, recurrent PE, idiopathic PE, DVT splenectomy, cancer, chronic inflammatory disease, pacemaker leads, thyreopathy, blood type other than " 0 ") are emerging robustly from international registries. In this study, we confirmed those well-known risk factors of CTEPH with representation similar to other studies. This means that the risk profile of patients with CTEPH in our country is comparable with knowledge about risk factors from other countries presented in up-to-date studies. The median of representativeness of risk factors is 3, with the most frequent combination of PE and/or recurrent PE, DVT, blood type other than "0".

Interestingly, the increased SPA together with the laboratory signs of higher platelet turnover was shown in patients with CTEPH, which could be a new possible predictive risk marker and a novel pathway in the pathogenesis of CTEPH. However, the value of this additional factor needs to be elucidated in further studies.

The prevalence of CTEPH in adult population in our country is estimated to be 1.8 per 100,000 inhabitants.

\section{Learning points}

Chronic thromboembolic pulmonary hypertension (CTEPH) is a progressive potentially fatal disease characterized by pulmonary obstruction caused by non-resolving but organizing thrombo-emboli combined with pulmonary vascular remodeling. The pathophysiology of CTEPH is not fully understood but the current knowledge from registries shows us that in order to prevent the development and progression of CTEPH, implement the effective therapeutic diagnostic strategies and decrease the mortality rates, it is crucial to identify the associated risk factors of pathophysiology. In our study, we approved multiple risk factors of CTEPH (previous PE, DVT, recurrent and idiopathic PE, thyreopathy, IBD, cancer, blood type other than " 0 ", splenectomy, coagulation abnormality, vWf, fVIII, PAI-1, fibrinogen). The multivariate of risk factors of CTEPH is explicit but we confirmed that the strongest combination is that of PE and/or recurrent PE, DVT, and blood type other than "0". Moreover, we identified platelet abnormalities (increased SPA and laboratory signs of higher platelet turnover) as a specific platelet behavior, which could be a potential new risk marker.

Additionally, we defined, that the prevalence of this rare disease in adult population from our country is 1.8 per 100,000 inhabitants. 


\section{References}

1. Galie N, Humbert M, Vachiery $\mathbf{J}$ et al. Guidelines for the diagnosis and treatment of pulmonary hypertension: The Joint Task Force for the Diagnosis and Treatment of Pulmonary Hypertension of the European Society of Cardiology (ESC) and the European Respiratory Society (ERS), endorsed by: Association for European Paediatric and Congenital Cardiology (AEPC), International Society for Heart and Lung Transplantation (ISHLT). Eur Heart J 2016; 37: 67-119. DOI: 10.1093/eurheartj/ehv317.

2. Hoeper MM, Madani MM, Nakanishi N et al. Chronic thromboembolic pulmonary hypertension. Lancet Respir Med 2014; 2: 573-582.

3. Lang IM, Pesavento R, Bonderman $D$ et al. Risk factors and basic mechanisms of chronic thromboembolic pulmonary hypertension: a current understanding. Eur Respir J 2013; 41: 462-468.

4. Lang IM. Chronic thromboembolic pulmonary hypertension - not so rare after all. N Engl J Med 2004; 350: 2236-2238.

5. Lang IM, Simonneau G, Pepke-Zaba JW et al. Factors associated with diagnosis and operability of chronic thromboembolic pulmonary hypertension. A case-control study. Thromb Haemost 2013; 110: 83-91.

6. Lang IM, Madani MM. Update on chronic thromboembolic pulmonary hypertension. Circulation 2014; 130: 508-518. DOI: 10.1161/CIRCULATIONAHA.114.009309.

7. Lang IM. Chronic thromboembolic pulmonary hypertension: a distinct disease entity. Eur Respir Rev 2015; 24: 246-252. DOI:10.1183/16000617.00001115.

8. Pepke-Zaba J, Delcroix M, Lang IM et al. Chronic Thromboembolic Pulmonary Hypertension (CTEPH): results from an International Prospective Registry. Circulation 2011; 124: 1973-1981.

9. Šimková I. CTEPH, Patofyziológia, klinický obraz, diagnostika. 199-215. In: Šimková I. Plúcna hypertenzia očami kardiológa. Bratislava; SAP, 2009.

10. Yang $\mathbf{S}$, Yang $\mathbf{Y}$, Zhai $\mathbf{Z}$ et al. Incidence and risk factors of chronic thromboembolic pulmonary hypertension in patients after acute pulmonary embolism J Thorac Dis 2015; 7 (11): 1927-1938. DOI: 10.3978/j.issn.20721439.2015.11.43.

11. Wong CL, Szydlo R, Gibbs S et al. Hereditary and acquired thrombotic risk factors for chronic thromboembolic pulmonary hypertension. Blood Coagul Fibrinolysis 2010; 21 (3): 201-206. DOI: 10.1097/ MBC.0b013e328331e664.

12. Dorfmüller P, Günther S, Ghigna MR et al. Microvascular disease in chronic thromboembolic pulmonary hypertension: a role for pulmonary veins and systemic vasculature. Eur Respir J. 2014; 44 (5): 1275-1288. DOI: 10.1183/09031936.00169113.

13. Condliffe R, Kiely DG, Gibbs JSR. Prognostic and aetiological factors in chronic thromboembolic pulmonary hypertension. Eur Resp J 2008; 33 (2): 332-338.

14. Bonderman D, Jakowitsch J, Adlbrecht $C$ et al. Medical conditions increasing the risk of chronic thromboembolic pulmonary hypertension. Thromb Haemost 2005; 93: 512-516.

15. Pepke-Zaba J, Jansa P, Kim NH et al. Chronic thromboembolic pulmonary hypertension: role of medical therapy. Eur Respir J 2013; 41: 985-990.

16. Franchini M. Hemostatic changes in thyroid diseases: haemostasis and thrombosis. Hematology. 2006; 11: 203-208.

17. Homoncik M, Gessl A, Ferlitsch A et al. Altered platelet plug formation in hyperthyroidism and hypothyroidism. J Clin Endocrinol Metab. 2007; 92: 3006-3012.

18. Bonderman $\mathrm{D}$, Wilkens $\mathrm{H}$, Wakounig $\mathrm{S}$ et al. Risk factors for chronic thromboembolic pulmonary hypertension. Eur Respir J 2009; 33: 325-331.
19. Frey MK, Alias S, Winter MP et al. Splenectomy Is Modifying the Vascular Remodeling of Thrombosis. J Am Heart Assoc 2014; 3: e000772. DOI: 10.1161/JAHA.113.000772.

20. Khan PN, Nair RJ, Olivares J et al. Postsplenectomy reactive thrombocytosis. Proc (Bayl Univ Med Cent) 2009; 22: 9-12.

21. Quarck R, Wynants M, Verbeken E. Contribution of inflammation and impaired angiogenesis to the pathobiology of chronic thromboembolic pulmonary hypertension Eur Respir J 2015; in press DOI: 10.1183/09031936.00009914.

22. Zabini D, Heinemann A, Foris $\mathbf{V}$ et al. Comprehensive analysis of inflammatory markers in chronic thromboembolic pulmonary hypertension patients. Eur Respir J 2014; 44: 951-962.

23. Wynants M, Quarck R, Ronisz A et al. Effects of C-reactive protein on human pulmonary vascular cells in chronic thromboembolic pulmonary hypertension. Eur Respir J 2012; 40: 886-894.

24. Bonderman D, Jakowitsch J, Adlbrecht $\mathbf{C}$ et al. Medical conditions increasing the risk of chronic thromboembolic pulmonary hypertension. Thromb Haemost 2005; 93: 512-516.

25. Kim NH, Lang IM. Risk factors for chronic thromboembolic pulmonary hypertension. Eur Respir Rev 2012; 21 (123): 27-31.

26. Bonderman D, Turecek PL, Jakowitsch $\mathbf{J}$ et al. High prevalence of elevated clotting factor VIII in chronic thromboembolic pulmonary hypertension. Thromb Haemost 2003; 90: 372-376.

27. Pengo V, Ruffatti A, Legnani $C$ et al. Incidence of a first thromboembolic event in asymptomatic carriers of high risk antiphospholipid antibody profile: a multicenter prospective study. Blood 2011; 118: 4714-4718.

28. Pengo V, Ruffatti A, Legnani $C$ et al. Clinical course of high-risk patients diagnosed with antiphospholipid syndrome. J Thromb Haemost 2010; 8: 237-242.

29. Koster T, Blann A.D, Briët $\mathbf{E}$ et al. Role of clotting factor VIII in effect of von Willebrand factor on occurrence of deep-vein thrombosis. Lancet. 1995; 345: 152-155.

30. Marsh JJ, Chiles PG, Liang N. Chronic thromboembolic pulmonary hypertension-associated dysfibrinogenemias exhibit disorganized fibrin structure. Thromb Res 132 (2013) 729-734. DOI: org/10.1016/j. thromres.2013.09.024.

31. Lang IM, Moser KM, Schleef RR. Elevated expression of urokinaselike plasminogen activator and plasminogen activator inhibitor type 1 during the vascular remodeling associated with pulmonary thromboembolism. Arterioscler Thromb Vasc Biol 1998; 18: 808-815.

32. Yaoita N, Shirakawa R, Fukumoto $\mathbf{Y}$ et al. Platelets Are Highly Activated in Patients of Chronic Thromboembolic Pulmonary Hypertension. Arterioscler Thromb Vasc Biol 2014; 34: 2486-2494. DOI: 10.1161/ATVBAHA.114.304404.

33. Remková A, Šimková I, Valkovičová T. Platelet abnormalities in chronic thromboembolic pulmonary hypertension. Int J Clin Exp Med 2015; 8 (6): 9700-9707.

34. Lindner J, Ambrož D, Novotný R et al. Pulmonary endarterectomy combined with cardiac surgery: A 7-year retrospective analysis. Cor Vasa 2015; 57 (2): e115-e120. DOI: http://dx.doi.org/10.1016/j.crvasa.2015.02.009.

35. Delcroix M, Lang IM, Pepke-Zaba J et al. Long-Term Outcome of Patients with Chronic Thromboembolic Pulmonary Hypertension: Results From an International Prospective Registry. Circulation 2016; 133 (9): 859-871.

Received March 24, 2016. Accepted May 9, 2016. 